\title{
Subcutaneous scalp nodule as the presenting symptom of systemic light-chain amyloidosis
}

\author{
Joseph Tadros ${ }^{1}$, Stacey Goodman ${ }^{2,3}$, Eric R. Tkaczyk ${ }^{2,3,4}$
}

\author{
1 University of Cincinnati College of Medicine, Cincinnati, OH, USA \\ 2 Vanderbilt University Medical Center, Nashville, TN, USA \\ 3 Department of Veterans Affairs, Tennessee Valley Health System, Nashville, TN, USA \\ 4 Department of Biomedical Engineering, Vanderbilt University, Nashville, TN, USA
}

\begin{abstract}
Key words: nodular amyloidosis, systemic AL amyloidosis, pilar cyst, subcutaneous nodule
Citation: Tadros J, Goodman S, Tkaczyk ER. Subcutaneous scalp nodule as the presenting symptom of systemic light-chain amyloidosis. Dermatol Pract Concept. 2018;8(3):184-187. DOI: https://doi.org/10.5826/dpc.0803a07
\end{abstract}

Received: October 1, 2017; Accepted: November 21, 2017; Published: July 31, 2018

Copyright: $@ 2018$ Tadros et al. This is an open-access article distributed under the terms of the Creative Commons Attribution License, which permits unrestricted use, distribution, and reproduction in any medium, provided the original author and source are credited.

Funding: None.

Competing interests: The authors have no conflicts of interest to disclose.

All authors have contributed significantly to this publication.

Corresponding author: Eric Tkaczyk, MD, PhD, Director, Vanderbilt Cutaneous Imaging Clinic, 719 Thompson Lane, Suite 26300, Nashville, TN 26300 USA. Email: eric.tkaczyk@vanderbilt.edu.

ABSTRACT We present a case of subcutaneous nodular amyloidosis mimicking a pilar cyst. Further evaluation led to the diagnosis of malignant systemic light-chain amyloidosis. The epidemiology and histopathological features of light-chain amyloidosis with cutaneous involvement are reviewed, as well as current recommendations for initial evaluation.

\section{Case Presentation}

An 81-year-old man with a history of carpal tunnel syndrome, diabetes mellitus, and nonmelanoma skin cancer presented with a 1-year history of an asymptomatic $4 \mathrm{~mm}$ diameter subcutaneous nodule on the right anterior scalp. A pilar cyst was suspected and an excisional biopsy was performed. Microscopic examination revealed extensive dermal infiltration by amorphous hyaline and fibrillary material (Figures 1-3) revealing the diagnosis of amyloidosis.

Initial laboratory workup revealed normal protein gap, unremarkable complete blood counts (CBC), and comprehensive metabolic panel. However, further laboratory investigation confirmed systemic light-chain (AL) amyloidosis involving several organ systems. Hematopoietic system involvement was characterized by an elevated serum free lambda light chain of $152 \mathrm{mg} / \mathrm{L}$ (range 5.71-26.30 mg/L), moderate serum lambda Bence-Jones protein, and faint urinary faint lambda Bence-Jones protein as measured by serum protein and urine protein electrophoresis. Cardiac involvement was evidenced by a right bundle branch block on electrocardiogram and moderate left ventricular hypertrophy with increased interventricular thickness and moderate diastolic dysfunction on echocardiogram. An underlying plasma cell dyscrasia was evidenced by findings on a bone marrow biopsy of a lambda-restricted increase in plasma cells (15\%-20\%) on immunohistochemistry and flow cytometry. Fortunately, a skeletal survey was negative, indicating no bony involvement. Additionally, autoimmune titers were within normal limits for antinuclear antibody (ANA), anti-Ro/SSA, and anti-La/ 


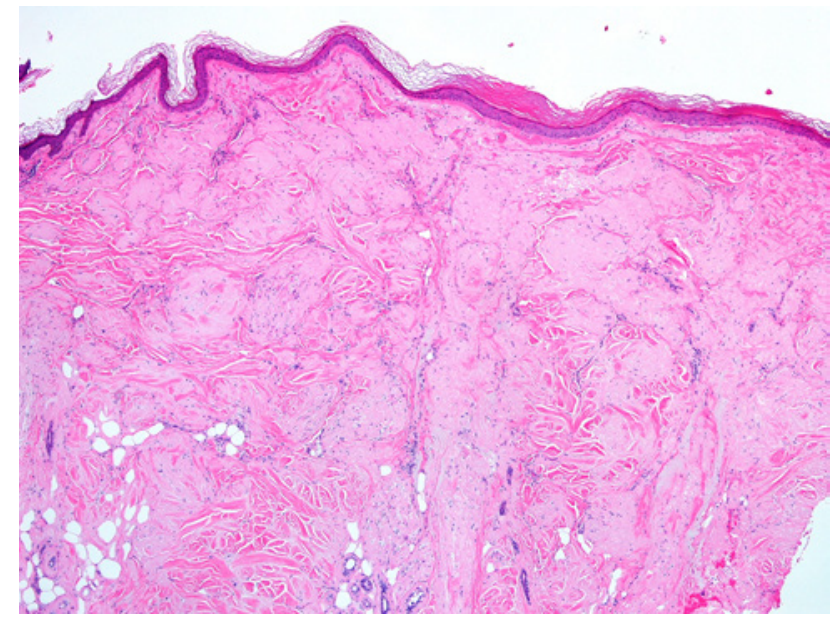

Figure 1. Amyloid deposition from excisional skin biopsy specimen (hematoxylin and eosin [H\&E] staining, original magnification $\times 4$ ). [Copyright: (C2018 Tadros et al.]

SSB autoantibodies. Diagnosis of systemic primary lambda light-chain amyloidosis with cutaneous manifestations was made. A cardiac MRI was obtained to further characterize the cardiac involvement. Treatment with cyclophosphamidebortezomib-dexamethasone was initiated with subsequent outpatient follow-up.

\section{Discussion}

Amyloidosis is not just one disease but a rare group of diseases resulting from the extracellular deposition of misfolded proteins in various tissues as toxic amyloid aggregates. Amyloidosis is characterized based on the subtype of amyloid fibril protein as well as the mechanism of deposition. Cutaneous amyloidosis may be organized into one of 3 groups: primary localized cutaneous amyloidosis (PLCA), secondary localized cutaneous amyloidosis, or systemic AL amyloidosis with cutaneous involvement [1]. Among the PLCA group, lichen, macular, biphasic, and nodular forms are recognized. The most encountered type of PLCA, lichen amyloidosis, presents clinically as multiple hyperpigmented, clustered, pruritic papules on the lower extremities [2]. Macular amyloidosis presents darkly pigmented macules with a rippled surface. Both lichen and macular types are derived from cytokeratins [2]. The biphasic form is simply a mix of the macular and lichen amyloidoses.

Nodular amyloidosis, accounting for $1.5 \%$ of PLCA cases, presents as either single or multiple lesions and is derived from immunoglobulins, commonly lambda light chain. There have been less than 100 cases of primary localized nodular amyloidosis reported to date [2-5]. Some studies suggest that nodular amyloidosis may be associated with systemic disease in $7 \%$ to $50 \%$ of patients $[6,7]$. However, a literature review conducted by Biewend et al found that

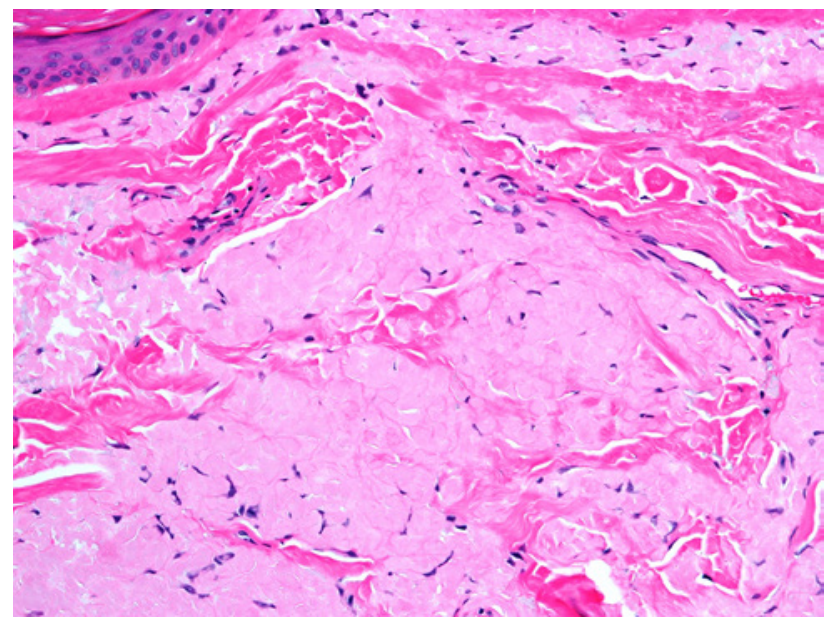

Figure 2. Amyloid deposition from excisional skin biopsy specimen (H\&E staining, original magnification $\times 40)$. Congo red stain revealed abundant eosinophilic, amorphous material exhibiting dermal apple-green birefringence with polarization, consistent with amyloid deposition (Figure 3). [Copyright: (C2018 Tadros et al.]

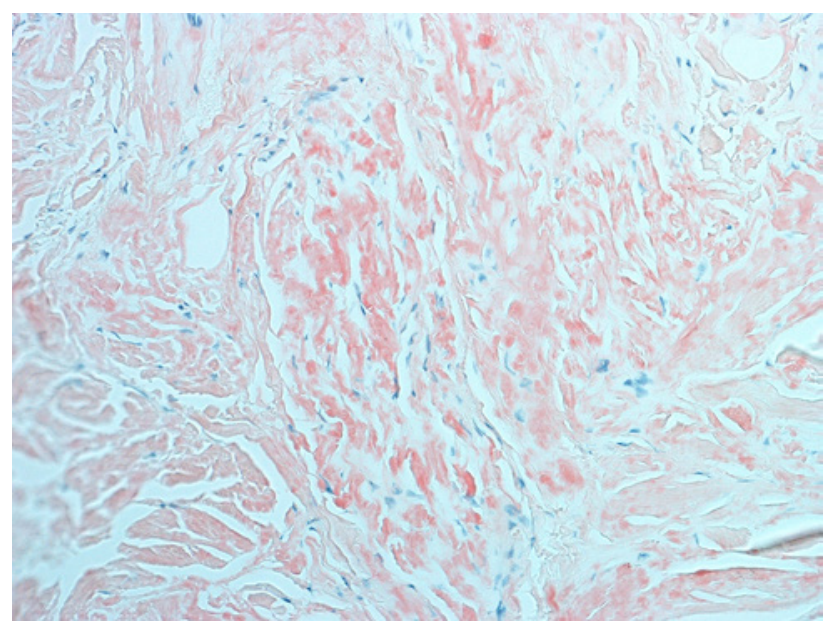

Figure 3. Amyloid deposition on excisional skin biopsy specimen (Congo red, original magnification $\times 40$ ). [Copyright: $@ 2018$ Tadros et al.]

of 290 PLCA cases at initial diagnosis, only 4 went on to develop systemic amyloidosis [3]. In their own retrospective institutional study identifying cases of localized nodular amyloidosis, 0 of the 20 patients went on to develop systemic disease over a 7.6-year mean follow-up period [3].

Though it can be PLCA, which is relatively benign and requires only routine monitoring, nodular amyloidosis can also be the presenting sign of a serious systemic pathology, such as systemic AL amyloidosis in this case. Systemic AL amyloidosis has a reported incidence of only 3 to 5 cases per million population, correlating to roughly 1750 to 3200 new cases annually in the United States [8,9]. Cutaneous involvement occurs in up to $29 \%$ to $40 \%$ of cases [10].

Histopathological examination of nodular amyloidosis is characterized by amyloid deposits, which appear as amorphous, acellular, fissured, eosinophilic material in the papil- 
lary and reticular dermis as well as in the subcutaneous fat [11]. Flattened rete ridges may be observed in the overlying epidermis. Amyloids stained with Congo red display applegreen birefringence under polarized light. Because nodular amyloid is produced by plasma cell infiltration, specimens may stain with immunohistochemical stains for kappa or lambda chains [11].

It is common for cysts to be falsely diagnosed based on appearance alone. One retrospective analysis aimed to see if histological findings correlated with clinical diagnosis. The authors found that 13 of 295 cases were positive for calcium deposition, described as calcinosis cutis, on histology [12]. These findings compromised all the cases under the deposition category: no cases of amyloid deposition were evident. This highlights the rarity of nodular amyloidosis mimicking a cystic lesion. Investigation of a benign-appearing subcutaneous nodule as the initial presentation for asymptomatic AL amyloidosis in a relatively healthy patient is atypical, as the diagnosis is often made after workup of unexplained cardiomyopathy, proteinuria, neuropathy, or hepatomegaly. It is difficult to determine the precise occurrence of this presentation given the limited number of cases in the literature.

Importantly, since nodular primary cutaneous amyloidosis is so rare, biopsy-confirmed amyloid lesions warrant further workup as immunoglobulin light-chain amyloid fibrils may be a complication of systemic and multiple myeloma-associated amyloidosis [13]. Therefore, evaluation for systemic involvement at the time of diagnosis is recommended. Plasma cell disorders are diagnosed by quantitative immunoglobulin assays, serum and urine free light chains, and serum protein electrophoresis or urine protein electrophoresis with immunofixation. Serum calcium and creatinine as well as a skeletal survey and bone marrow biopsy are used to better characterize the plasma cell dyscrasias. The diagnosis of active multiple myeloma requires greater than $10 \%$ clonal plasma cells on bone marrow biopsy plus one or more multiple myeloma defining events based on the CRAB criteria: hypercalcemia, renal failure, anemia, or lytic bone lesions. In this context, our patient had inactive myeloma-associated AL amyloidosis.

Presenting clinical symptoms of systemic AL amyloidosis varies based on the organ system affected. Cardiac involvement may manifest as a new AV or bundle branch block; renal involvement by albuminuria from glomerular injury; GI involvement by nausea, vomiting, or diarrhea; autonomic dysfunction by symptoms of orthostasis or GI dysmolity; and neurologic involvement by peripheral neuropathy. Initial workup for systemic amyloidosis may include CBC, chemistries, serum free light chains, serum and urine protein electrophoresis with immunofixation, and urinary Bence-Jones protein [14]. The presence of a monoclonal protein is found in $80 \%$ to $90 \%$ of serum and urine immunochemistry studies in patients with primary amyloidosis [15]. Bone marrow biopsy is done to monitor for monoclonal plasma cells and if present, requires further evaluation to determine whether the patient has active multiple myeloma [15]. Aspiration of subcutaneous tissues of the abdominal wall or of an involved organ (in our patient, the skin) is required to definitively confirm the diagnosis of systemic amyloidosis [16]. Because primary nodular amyloidosis has a significant association with Sjögren syndrome, serology for ANA, anti-Ro/SSA, and anti-La/SSB may also be considered, which in this case were unremarkable [17-19].

\section{Conclusions}

Subcutaneous nodules, which may appear clinically benign, should not be ignored; physicians must consider the patient's history when assessing these lesions. The incidental finding of amyloid deposition on biopsy of a subcutaneous nodule in an asymptomatic, healthy individual warrants further evaluation to better determine if it represents localized amyloidosis or the initial manifestation of a systemic process. Although a rare initial presentation, cutaneous manifestations of systemic AL amyloidosis may pose as the first clue toward successful diagnosis.

\section{Acknowledgement}

Dr. Tkaczyk is grateful for the support from NIH K12 CA 090625 .

\section{References}

1. Schucht K, Schröder J, Siegmund H, Grafe C, Schreml S. Nodular cutaneous amyloidosis at the temple. Case Rep Dermatol. 2016;8(2):193-196. doi: 10.1159/000447234.

2. Weidner T, Illing T, Elsner P. Primary localized cutaneous amyloidosis: a systematic treatment review. Am J Clin Dermatol. 2017;18(5):629-642. doi: 10.1007/s40257-017-0278-9.

3. Kaltoft B, Schmidt G, Lauritzen AF, Gimsing P. Primary localised cutaneous amyloidosis—a systematic review. Dan Med J. 2013;60(11):A4727.

4. Biewend ML, Menke DM, Calamia KT. The spectrum of localized amyloidosis: a case series of 20 patients and review of the literature. Amyloid. 2006;13(3):135-142. doi: 10.1080/13506120600876773.

5. Nguyen TU, Oghalai JS, McGregor DK, Janssen NM, Huston DP. Subcutaneous nodular amyloidosis: a case report and review of the literature. Hum Pathol. 2001;32(3):346-348. doi: 10.1053/ hupa.2001.22742.

6. Brownstein MH, Helwig EB. The cutaneous amyloidoses. I. Localized forms. Arch Dermatol. 1970;102(1):8-19. doi: 10.1001/ archderm.1970.04000070010002.

7. Woollons A, Black MM. Nodular localized primary cutaneous amyloidosis: a long-term follow-up study. Br J Dermatol. 2001;145(1):105-109. doi: 10.1046/j.1365-2133.2001.04291.x.

8. Wechalekar AD, Gillmore JD, Hawkins PN. Systemic amyloidosis. Lancet. 2016;387(10038):2641-2654. doi: 10.1016/S01406736(15)01274-X. 
9. Falk RH, Comenzo RL, Skinner M. The systemic amyloidoses. N Engl J Med. 1997;337(13):898-909. doi: 10.1056/ NEJM199709253371306.

10. Steciuk A, Dompmartin A, Troussard X, et al. Cutaneous amyloidosis and possible association with systemic amyloidosis. Int J Dermatol. 2002;41(3):127-132; discussion 133-124. doi: 10.1046/j.1365-4362.2002.01411.x.

11. Borrowman TA, Lutz ME, Walsh JS. Cutaneous nodular amyloidosis masquerading as a foot callus. J Am Acad Dermatol. 2003;49(2):307-310. doi: 10.1067/S0190-9622(03)00433-X.

12. Ring CM, Kornreich DA, Lee JB. Clinical simulators of cysts. J Am Acad Dermatol. 2016;75(6):1255-1257. doi: 10.1016/j. jaad.2016.07.013.

13. Breathnach SM. The cutaneous amyloidoses. Pathogenesis and therapy. Arch Dermatol. 1985;121(4):470-475. doi: 10.1001/ archderm.1985.01660040054011.

14. Musbahi E, Hameed O, Lateo S. A case presentation of nodular primary localized cutaneous amyloidosis: learning points and update. J Am Acad Dermatol. 2015;72(5)(suppl 1):AB36. doi: 10.1016/j.jaad.2015.02.155.
15. Wei A, Juneja S. Bone marrow immunohistology of plasma cell neoplasms. J Clin Pathol. 2003;56(6):406-411. doi: 10.1136/ jcp.56.6.406.

16. van Gameren II, Hazenberg BP, Bijzet J, van Rijswijk MH. Diagnostic accuracy of subcutaneous abdominal fat tissue aspiration for detecting systemic amyloidosis and its utility in clinical practice. Arthritis Rheum. 2006;54(6):2015-2021. doi: 10.1002/ art.21902.

17. Vitali C, Bombardieri S, Jonsson R, et al; European Study Group on Classification Criteria for Sjögren's Syndrome. Classification criteria for Sjögren's syndrome: a revised version of the European criteria proposed by the American-European Consensus Group. Ann Rheum Dis. 2002;61(6):554-558. doi: 10.1136/ ard.61.6.554.

18. Brown AJ, Spicknall KE, Mutasim DF. Multiple lesions of primary cutaneous nodular amyloidosis in Sjögren syndrome. $J$ Am Acad Dermatol. 2012;67(6):e267-e268. doi: 10.1016/j. jaad.2012.05.012.

19. Butler J. Sjögren syndrome-associated localized cutaneous nodular amyloidosis. J Am Acad Dermatol. 2013;68(4)(suppl 1):AB72. 